\title{
Analysis of Friction Coupling and the Painlevé Paradox in Multibody Systems
}

\author{
Albert Peiret · József Kövecses · Josep M. Font-Llagunes
}

Received: date / Accepted: date

\begin{abstract}
Multibody models are useful to describe the macroscopic motion of the elements of physical systems. Modelling contact in such systems can be challenging, especially if friction at the contact interface is taken into account. Furthermore, the dynamics equations of multibody systems with contacts and Coulomb friction may become ill-posed due to friction coupling, as in the Painlevé paradox, where a solution for system dynamics may not be found. Here, the dynamics problem is considered following a general approach so that friction phenomena, such as dynamic jamming, can be analyzed. The effect of the contact forces on the velocity field of the system is the cornerstone of the proposed formulation, which is used to analyze friction coupling in multibody systems with a single contact. In addition, we introduce a new representation of the so-called generalized friction cone, a quadratic form defined in the contact velocity space. The geometry of this cone can be used to determine the critical cases where the solvability of the system dynamic equations can be compromised. Moreover, it allows for assessing friction coupling at the contact interface, and obtain the values of the friction coefficient that can make the dynamics formulation inconsistent. Finally, the classical Pailevé example of a single rod and the multibody model of an articulated arm are used to illustrate how the proposed cone can detect the cases where the dynamic equations have no solution, or multiple solutions.
\end{abstract}

\section{Introduction}

Modelling, analyzing, and simulating multibody systems with contacts are ongoing research topics. Considering friction at the contact interface increases the complexity of the model, thus presenting some challenges, especially when the solution of the dynamic equations is required. The Coulomb friction model is very representative for modelling contact, and is able to describe the macroscopic motion of the bodies in the system, which is convenient in rigid multibody systems. Moreover, this model is well-known for capturing phenomena related to friction, such as the stick-slip transition [1]. However, the use of Coulomb friction can make the dynamic equations have no solution or several, under some circumstances, which gives rise to the Painlevé paradox [2,3].

In the absence of friction, contact can be modelled with unilateral constraints. In such a case, the dynamic formulation with frictionless contacts leads to a linear complementarity problem (LCP) [4], for which the existence of solution is guaranteed [5] and various solver algorithms are available [6, 7,8]. On the other hand, if unilateral

A. Peiret · J. Kövecses

Department of Mechanical Engineering and Centre for Intelligent Machines, McGill University, 817 Sherbrooke St. West, Montreal, H3A 0C3, Québec, Canada

E-mail: albert.peiret@mail.mcgill.ca

E-mail: jozsef.kovecses@mcgill.ca

J. M. Font-Llagunes

Biomechanical Engineering Lab, Department of Mechanical Engineering and Research Centre for Biomedical Engineering, Universitat Politècnica de Catalunya, Av. Diagonal 647, 08028 Barcelona, Catalunya, Spain

E-mail: josep.m.font@upc.edu 
constraints with Coulomb friction are used to model the contact interaction, the formulation in general leads to a nonlinear complementarity problem (NCP) [9]. The solution of such problems cannot be guaranteed in a systematic way, and there are not as many solver algorithms available as for LCP problems. Faceted (or polyhedral) approximations of the friction cone can be used to lead the model back to an LCP problem. These can be classified into two main approaches: velocity discretization [10,11], and force discretization [12,13] methods. The solvability of these formulations may be compromised in some circumstances $[14,15,16]$, where there is no solution for the forces and accelerations of the system. Nevertheless, it is possible to formulate the equations at the velocity level by introducing a finite difference approximation of the accelerations, so that a solution for the velocities and impulses can always be found [12].

Some authors have proposed formulations for non-smooth systems that rely on the maximum dissipation principle $[17,18,19]$. In some cases, this makes it possible to formulate the impulse-momentum dynamics as a convex optimization problem [17] or a monotone optimization problem [19]. However, if there is friction coupling, i.e., the contact normal and tangent directions are coupled to each other, then the contact problem with Coulomb friction leads to non-convex optimization problems. To overcome this, the problem can be convexified by using the dual of the friction cone [20,21]. The maximum dissipation principle was also used in [22] to derive a generalized fiction law, which can cope with the indeterminacy problem of Pailevé-like examples by choosing one solution from all the possibles, although the solution might not be unique.

Friction in sliding contacts can cause a phenomenon known as dynamic jamming (or locking) [23, 24, 25], which is very closely related to the jamb process in collisions [26,27]. Essentially, due to friction coupling, the kinetic friction force can generate a compression of the contact interface, similar to an impact but without a collision, which leads to an abrupt change in the velocities $[9,25,28]$. Depending on how the system dynamics is formulated, it is not possible to find a solution for the contact force, therefore making it impossible to determine the system acceleration. To resolve this problem, the dynamic equations can be reformulated at the impulsemomentum level, so that the system velocity right after the locking process can be determined [24, 29]. However, it is also possible to characterize the system compliance at the contact interface, which allows it to compress and develop the required contact force [30]. Then, the time integration can continue and the system dynamics can be formulated as desired [31,32].

The friction cone arises from the fact that the Coulomb model limits the magnitude of the static friction force; consequently, the resultant contact force vector of a non-sliding contact must lie within the cone. Moreover, the contact force of a sliding contact must lie on the surface of the cone, because the magnitude of the friction force is proportional to the normal force component. On the other hand, the generalized friction cone is a concept that goes beyond the contact forces and takes the system dynamics into account. Erdmann [33] introduced the generalized friction cone for one rigid body with planar motion, which was used to describe the effect of the contact forces onto the generalized coordinates and velocities. In addition, he noted that the dynamic equations may become inconsistent when the cone dips below the tangent plane. Similarly, Génot and Brogliato [28] reported that the shape of the generalized friction cone is directly related to the existence of solution of the dynamic formulation for the so-called Painlevé example. Interestingly, the shape of this cone depends not only on the friction coefficient, but also on the configuration and mass distribution of the system. However, it is defined in the multi-dimensional velocity space, and so the visualization of that cone is not always possible, except for simple cases with one body in planar motion $[28,33]$.

In this paper, we propose a new representation of the generalized friction cone that is defined in the contact space as opposed to the velocity space of the system. The contact space is a 3-dimensional space for one contact point in a system with general spatial motion, which helps to visualize the cone and allows us to analyze the effect of friction coupling in multibody systems. The implicit equation of the cone for one contact point is derived and analyzed. Moreover, the limit value of the friction coefficient for which the dynamic equations of the system can become inconsistent is obtained analytically. The dynamics of systems with contact and friction is discussed with a general approach, and the existence of solution is assessed for several examples. 


\section{Dynamics of Multibody Systems with Contact}

Let $\mathbf{v}$ be the array of the $n$ generalized velocities of a multibody system, and $\mathbf{q}$ the set of $p$ generalized coordinates, such that

$$
\dot{\mathbf{q}}=\mathbf{N v}
$$

where $\mathbf{N}(\mathbf{q})$ is the $p \times n$ transformation matrix that depends on the parametrization of both the configuration and velocity of the system. This definition makes the approach more general, because the elements of $\mathbf{q}$ and $\mathbf{v}$ can also be defined using non-holonomic velocity components. For instance, if Euler parameters are used as generalized coordinates, and angular velocity components as generelaized velocities [34]. Therefore, $p \geqslant n$ in general.

The components of $\mathbf{v}$ parametrize the tangent space that locally represents the configuration space defined by q. Then, the dynamic equations that govern the system associated with the generalized velocities $\mathbf{v}$ are

$$
\mathbf{M} \dot{\mathbf{v}}+\mathbf{c}=\mathbf{f}
$$

where $\mathbf{M}(\mathbf{q})$ is the $n \times n$ positive-definite mass matrix, $\mathbf{c}$ contains the Coriolis and centrifugal terms, and $\mathbf{f}$ represents internal and external generalized forces.

Interaction through contact is commonly modelled as force vectors, which are applied on one or several contact points, depending on the level of complexity of the interacting surfaces. By choosing a set of contact points, the array of generalized forces can be expressed as

$$
\mathbf{f}=\mathbf{f}_{0}+\mathbf{A}^{\mathrm{T}} \boldsymbol{\lambda}_{\mathrm{c}}
$$

where $\mathbf{f}_{0}$ contains the generalized forces of the other known forces and torques (external or internal), and other interactions. $\boldsymbol{\lambda}_{\mathrm{c}}$ are the $r$ contact force components of the contacts considered, and $\mathbf{A}(\mathbf{q})$ is the $r \times n$ contact Jacobian matrix that maps the generalized velocities to the contact velocity components

$$
\mathbf{u}_{\mathrm{c}}=\mathbf{A v}
$$

This definition of the generalized contact forces is general for any number of contacts, and also for contacts with friction, because the components in $\boldsymbol{\lambda}_{\mathrm{c}}$ can represent both normal and friction force components as it is shown below. These $r$ force components represent the contact interaction, and for one contact point with friction, one normal component and two tangential components are needed. This section focuses on the effect that contact interactions have on the dynamics of a multibody system, which will be used to analyze contacts with Coulomb friction later on.

Generally, the force components can be defined via either constitutive relations or kinematic constraints. By using a constitutive relation, the force components $\boldsymbol{\lambda}_{\mathrm{c}}=\boldsymbol{\lambda}_{\mathrm{c}}(\mathbf{q}, \mathbf{v}, t)$ are fully given by the state of the system (and other state variables of the contact model, such as in bristle-based friction models [35]). On the other hand, the force components $\boldsymbol{\lambda}_{\mathrm{c}}$ become unknowns of the dynamic equations if the contact velocities are constrained, so that $\mathbf{u}_{\mathrm{c}}=\mathbf{b}(\mathbf{q}, t)$ for bilateral contacts, or $\mathbf{u}_{\mathrm{c}} \geqslant \mathbf{b}(\mathbf{q}, t)$ for unilateral contacts; and usually $\mathbf{b} \equiv \mathbf{0}$. Nevertheless, to keep a general approach, we will not make a distinction here between force definition via constitutive relations or kinematic constraints.

The infinitesimal change in the system velocity vector is given by (2) and (3) as

$$
\mathrm{d} \mathbf{v}=\delta \mathbf{v}_{\mathrm{c}}+\delta \mathbf{v}_{0}
$$

where

$$
\begin{aligned}
\delta \mathbf{v}_{\mathrm{c}} & =\mathbf{M}^{-1} \mathbf{A}^{\mathrm{T}} \boldsymbol{\lambda}_{\mathrm{c}} \mathrm{d} t \\
\delta \mathbf{v}_{0} & =\mathbf{M}^{-1}\left(\mathbf{f}_{0}-\mathbf{c}\right) \mathrm{d} t
\end{aligned}
$$

are the contribution of the contact forces $\boldsymbol{\lambda}_{\mathrm{c}}$ and all the other forces to the total velocity change dv, respectively. It is important to mention that the notation " $\mathrm{d} x$ " is used for the infinitesimal change in the variable " $x$ ", while " $\delta y$ " only indicates a contribution to an infinitesimal change, and therefore, " $y$ " does not necessarily exist.

Contact forces $\boldsymbol{\lambda}_{\mathrm{c}}$ only affect a certain component of the total velocity increment according to (6), which is confined in a subspace spanned by the columns of $\mathbf{M}^{-1} \mathbf{A}^{\mathrm{T}}$. The dimension of this subspace is equal to the rank 


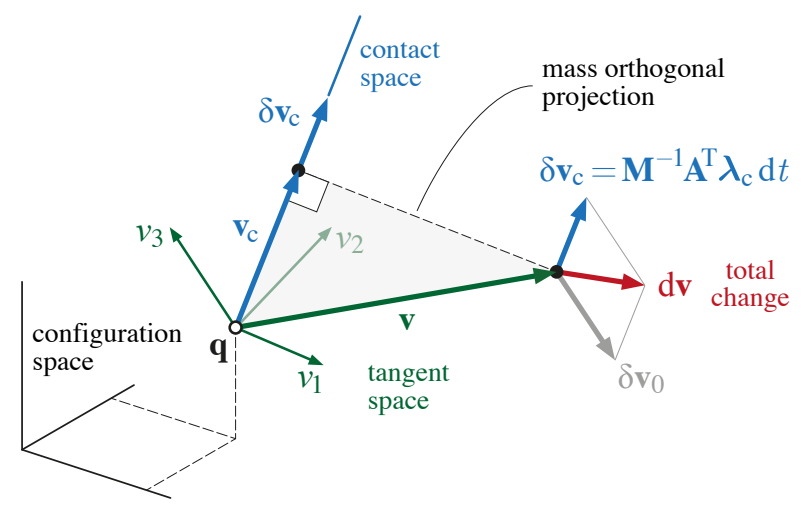

Fig. 1 Illustration of the configuration space $\mathbf{q}$, and the velocity space parametrized by $\mathbf{v}=\left[v_{1}, v_{2}, v_{3}\right]^{\mathrm{T}}$, where $n=3$ only as an example. Note that the contribution of the contact forces $\boldsymbol{\lambda}_{\mathrm{c}}$ to the change in the system velocity $\mathbf{v}$ only affects the velocity component in the contact space $\mathbf{v}_{\mathrm{c}}=\mathbf{P}_{\mathrm{c}} \mathbf{v}$.

of the Jacobian matrix A since the mass matrix $\mathbf{M}$ is full rank. Moreover, it is possible to decompose the system velocity into two orthogonal complement subspaces of the tangent space [36], so that the contact forces only affect the dynamics of the system in one of them, which shall be called contact space (see Fig. 1).

The component of the generalized velocity vector in the contact space can be defined via the projection

$$
\mathbf{v}_{\mathrm{c}}=\mathbf{P}_{\mathrm{c}} \mathbf{v}
$$

where the idempotent projector matrix is

$$
\mathbf{P}_{\mathrm{c}}=\mathbf{M}^{-1} \mathbf{A}^{\mathrm{T}}\left(\mathbf{A} \mathbf{M}^{-1} \mathbf{A}^{\mathrm{T}}\right)^{-1} \mathbf{A}
$$

which can be computed straightforwardly if there is no redundancy in the contact force components, i.e., $\mathbf{A}$ is full row rank [36]. Otherwise, if the contact forces are redundant, a set of independent rows of $\mathbf{A}$ could be selected to compute the projector matrix. Other numerical techinques can also be used, but the properties associated with this projection would still hold in any case [36]. This projector operator allows us to decompose the system velocity to two components

$$
\mathbf{v}=\mathbf{v}_{\mathrm{c}}+\mathbf{v}_{\mathrm{a}}
$$

where $\mathbf{v}_{\mathrm{a}}=\left(\mathbf{I}_{n}-\mathbf{P}_{\mathrm{c}}\right) \mathbf{v}$ is the orthogonal complement of the velocity component in the contact space $\mathbf{v}_{\mathrm{c}}$, and $\mathbf{I}_{n}$ is the $n \times n$ identity matrix. This decomposition allows us to decouple the dynamics of the system in these two subspaces. Here, only the contact space is relevant for our purpose, because it is the only one affected by the contact dynamics.

The total change in the velocity can be decomposed as

$$
\mathrm{d} \mathbf{v}=\mathrm{d} \mathbf{v}_{\mathrm{c}}+\mathrm{d} \mathbf{v}_{\mathrm{a}}=\mathbf{P}_{\mathrm{c}} \mathrm{d} \mathbf{v}+\left(\mathbf{I}_{n}-\mathbf{P}_{\mathrm{c}}\right) \mathrm{d} \mathbf{v}
$$

where

$$
\mathrm{d} \mathbf{v}_{\mathrm{c}}=\delta \mathbf{v}_{\mathrm{c}}+\mathbf{P}_{\mathrm{c}} \delta \mathbf{v}_{0}
$$

which contains the change in velocity due to the contact force $\delta \mathbf{v}_{\mathrm{c}}$ in Eq. (6). It can be shown that the projection matrix $\mathbf{P}_{\mathrm{c}}$ does not affect this component, i.e., $\delta \mathbf{v}_{\mathrm{c}}=\mathbf{P}_{\mathrm{c}} \delta \mathbf{v}_{\mathrm{c}}$. Therefore, the contact forces only affect the velocity component in the contact space $\mathbf{v}_{\mathrm{c}}$, as illustrated in Fig. 1.

The contact space represents the components of the velocity field of the system that are affected by the contact forces. Since the velocity field of the system can be parametrized by the generalized velocities $\mathbf{v}$, one possible parametrization of the contact space is given by the component $\mathbf{v}_{\mathrm{c}}$. The contact velocity components $\mathbf{u}_{\mathrm{c}}$ can also parametrize the contact space given the relation

$$
\mathbf{v}_{\mathrm{c}}=\mathbf{P}_{\mathrm{c}} \mathbf{v}=\mathbf{M}^{-1} \mathbf{A}^{\mathrm{T}}\left(\mathbf{A} \mathbf{M}^{-1} \mathbf{A}^{\mathrm{T}}\right)^{-1} \mathbf{u}_{\mathrm{c}}
$$

where the relation $\mathbf{u}_{\mathrm{c}}=\mathbf{A v}$ has been used. Therefore, the contact velocity $\mathbf{u}_{\mathrm{c}}$ provides a parametrization that can be used to represent the system dynamics in the contact space [36]. Moreover, the $r$ contact velocity components in 
$\mathbf{u}_{\mathrm{c}}$ are independent from each other if the contact forces are not redundant. However, the $n$ generalized velocities in $\mathbf{v}_{\mathrm{c}}$ are dependent as long as the contact space is smaller than the tangent space (i.e., $\mathbf{P}_{\mathrm{c}} \neq \mathbf{I}_{n}$ ).

Likewise, the change in the contact velocities is given by

$$
\mathrm{d} \mathbf{u}_{\mathrm{c}}=\delta \mathbf{u}_{\mathrm{c}}+\delta \mathbf{u}_{0}
$$

where

$$
\begin{aligned}
& \delta \mathbf{u}_{\mathrm{c}}=\mathbf{A} \mathbf{M}^{-1} \mathbf{A}^{\mathrm{T}} \boldsymbol{\lambda}_{\mathrm{c}} \mathrm{d} t \\
& \delta \mathbf{u}_{0}=\left(\mathbf{A} \mathbf{M}^{-1}\left(\mathbf{f}_{0}-\mathbf{c}\right)+\dot{\mathbf{A}} \mathbf{v}\right) \mathrm{d} t
\end{aligned}
$$

are the contribution to the change in the contact velocities $\mathbf{u}_{c}$ of the contact forces $\boldsymbol{\lambda}_{\mathrm{c}}$, and the contribution of the rest of the forces acting on the system, respectively. Note that $\dot{\mathbf{A}}$ denotes the time derivative of the contact Jacobian matrix. The idea that the system dynamics can be projected to a subspace parametrized by the contact velocities $\mathbf{u}_{\mathrm{c}}$ makes it possible to study the dynamics of multibody systems with contacts only in the contact space, which is used below to analyze systems with contact and Coulomb friction.

As reported in the literature [2,3,5,9,25,28,30,31,32], paradoxical situations may arise when Coulomb friction is used in models of rigid bodies, which can even happen in systems of only one body in planar motion and one single contact point. In this paper, we aim to illustrate these phenomena for more complex multibody systems with motion in 3-dimensional space and a single contact point. Nonetheless, the analysis performed can be extended to systems with multiple contact points by taking the appropriate considerations into account. For instance, if contact is modelled via constraints, the analysis of one of the contact points can be done by projecting the dynamics of the multibody system into a subspace that is not affected by the contact forces at the other points, as explained in [36].

\section{Contact with Coulomb Friction}

The interaction between bodies through frictional contact can be described by the Coulomb model, which defines the contact force components based on a non-smooth friction law. The force acting on each contact point can be decomposed as

$$
\boldsymbol{\lambda}_{\mathrm{c}}=\left[\begin{array}{l}
\boldsymbol{\lambda}_{\mathrm{t}} \\
\lambda_{\mathrm{n}}
\end{array}\right]
$$

where $\lambda_{\mathrm{t}}=\left[\begin{array}{ll}\lambda_{\mathrm{t} 1} & \lambda_{\mathrm{t} 2}\end{array}\right]^{\mathrm{T}} \in \mathbb{R}^{2}$ are the tangential force components along two orthogonal directions in the tangent plane, which are associated with friction, and $\lambda_{\mathrm{n}} \in \mathbb{R}$ is the normal force component. Accordingly, the contact velocity of each contact point in a multibody system can be parametrized be the normal velocity component $u_{\mathrm{n}} \in \mathbb{R}$ and the two tangential components in $\mathbf{u}_{\mathrm{t}}=\left[\begin{array}{ll}u_{\mathrm{t} 1} & u_{\mathrm{t} 2}\end{array}\right]^{\mathrm{T}} \in \mathbb{R}^{2}$ (or sliding velocity). Which can be arranged in matrix form as

$$
\mathbf{u}_{\mathrm{c}}=\left[\begin{array}{l}
\mathbf{u}_{\mathrm{t}} \\
u_{\mathrm{n}}
\end{array}\right]=\left[\begin{array}{l}
\mathbf{A}_{\mathrm{t}} \\
\mathbf{A}_{\mathrm{n}}
\end{array}\right] \mathbf{v}=\mathbf{A} \mathbf{v}
$$

The Coulomb friction model defines the friction force with two phases: static and kinetic. In the static phase, friction acts as a constraint to prevent the contact point from sliding, i.e., $\mathbf{u}_{\mathbf{t}}=\mathbf{0}$. However, the magnitude of the static friction force is limited by the static friction coefficient $\mu_{\mathrm{s}}$ as

$$
\left\|\lambda_{\mathrm{t}}\right\|=\sqrt{\boldsymbol{\lambda}_{\mathrm{t}}^{\mathrm{T}} \boldsymbol{\lambda}_{\mathrm{t}}}<\mu_{\mathrm{s}} \lambda_{\mathrm{n}}
$$

On the other hand, the kinetic friction force always opposes the sliding velocity, and can be defined as

$$
\lambda_{\mathrm{t}}=-\mu_{\mathrm{k}} \lambda_{\mathrm{n}} \mathbf{e}_{\mathrm{t}}
$$

where $\mu_{\mathrm{k}}$ is kinetic friction coefficient. The sliding direction is defined by the unit vector $\mathbf{e}_{\mathrm{t}}=\frac{\mathbf{u}_{\mathrm{t}}}{\left\|\mathbf{u}_{\mathrm{t}}\right\|} \in \mathbb{R}^{2}$, which is the direction of the sliding velocity vector $\mathbf{u}_{\mathrm{t}}$.

Equation (19) defines the region inside the classic friction cone, in which the contact force $\boldsymbol{\lambda}_{\mathrm{c}}$ is contained, if the contact is not sliding. This cone becomes part of the dynamics formulation to establish the bounds of the static 
friction force. When unilateral constraints are used to define the normal contact force, an NCP problem can be formulated. Nevertheless, faceted approximations of the friction cone can make the equations formulate an LCP problem $[11,12,13]$.

On the other hand, all possible kinetic friction forces given by (20) generate a conic surface parametrized by the normal force $\lambda_{\mathrm{n}}$ and the sliding direction $\mathbf{e}_{\mathrm{t}}$, on which all the possible contact forces lie. Even though the direction of the kinetic friction force is determined by a given sliding velocity, looking at all the possible directions of the contact force as a conic surface can help to gain insight into the friction phenomenon, as it will be shown below. Therefore, the classic friction cone $\kappa_{\mu}$ can be defined as the region inside the conic surface given by

$$
\kappa_{\mu}\left(\boldsymbol{\lambda}_{\mathrm{c}}\right)=\boldsymbol{\lambda}_{\mathrm{c}}^{\mathrm{T}} \mathbf{Q}_{\mu} \boldsymbol{\lambda}_{\mathrm{c}}=\left[\begin{array}{l}
\boldsymbol{\lambda}_{\mathrm{t}} \\
\boldsymbol{\lambda}_{\mathrm{n}}
\end{array}\right]^{\mathrm{T}}\left[\begin{array}{cc}
\mathbf{I}_{2} & \mathbf{0} \\
\mathbf{0} & -\mu^{2}
\end{array}\right]\left[\begin{array}{l}
\lambda_{\mathrm{t}} \\
\lambda_{\mathrm{n}}
\end{array}\right]=0
$$

where $\mathbf{I}_{2}$ is the $2 \times 2$ identity matrix, and $\mu$ is the friction coefficient ( $\mu_{\mathrm{s}}$ or $\mu_{\mathrm{k}}$ for static or kinetic friction, respectively).

\section{The Generalized Friction Cone}

To analyze the effect of Coulomb friction in multibody systems, a similar concept to the friction cone $\kappa_{\mu}$ can be considered. The generalized friction cone $\kappa_{\mathrm{g}}$ for a contact point takes the dynamics of the system into account and describes the effect of the contact forces on the change in velocity of the system. The contribution to the change in the generalized velocities in Eq. (6) due to contact forces in the friction cone is

$$
\delta \mathbf{v}_{\mathrm{c}}=\mathbf{M}^{-1} \mathbf{A}^{\mathrm{T}} \boldsymbol{\lambda}_{\mathrm{c}} \mathrm{dt}, \quad \boldsymbol{\lambda}_{\mathrm{c}} \in \mathbb{R}^{3}: \kappa_{\mu}\left(\boldsymbol{\lambda}_{\mathrm{c}}\right) \leqslant 0
$$

which gives a parameterization of the generalized friction cone in the velocity space [28]. For each contact point, by using the Coulomb law in Eq. (20), the surface of the cone can be parametrized by the two friction force components $\lambda_{\mathrm{t}} \in \mathbb{R}^{2}$ as

$$
\delta \mathbf{v}_{\mathrm{c}}\left(\boldsymbol{\lambda}_{\mathrm{t}}\right)=\mathbf{M}^{-1}\left(\mathbf{A}_{\mathrm{t}}^{\mathrm{T}} \boldsymbol{\lambda}_{\mathrm{t}}+\frac{1}{\mu} \mathbf{A}_{\mathrm{n}}^{\mathrm{T}} \sqrt{\boldsymbol{\lambda}_{\mathrm{t}}^{\mathrm{T}} \boldsymbol{\lambda}_{\mathrm{t}}}\right) \mathrm{d} t
$$

for any $\mu>0$. Here, either the kinetic or static friction coefficients can be used, depending on whether or not the contact is sliding. The parameterization in Eq. (23) generates a conic surface with every possible $\delta \mathbf{v}_{\mathrm{c}}$ associated with all the contact forces $\lambda_{c}$ that satisfy Eq. (21). In case of kinetic friction $\delta \mathbf{v}_{c}$ lies on the surface of the cone, whereas for static friction $\delta \mathbf{v}_{\mathrm{c}}$ lies in the region inside the cone.

This cone lives in the tangent space, which is an $n$-dimensional space, and so its representation can be challenging. In order to visualize the cone, it can be projected onto a subspace. However, some information may be lost in the projection if the contact space is not contained in that subspace. This is because, the total effect of the contact forces $\delta \mathbf{v}_{\mathrm{c}}$ is contained in the contact space, see Eq. (12). For intance in [28], the velocity space of the system with one rigid body in planar motion has a dimension $n=3$, but the generalized cone is represented using only two velocities: the vertical velocity component, and the angular velocity. In that representation, it is not possible to see the effect of the contact force on the horizontal velocity, and this information cannot be visualized. Although, the contact space has dimension $r=2$, since the contact is in planar motion, the total effect of the contact forces is not represented. This might not be a problem for such simple examples, but this issue can be difficult to address for problems with a larger number of degrees of freedom.

Here, we perform a meaningful projection of the generalized cone onto the contact space, so that it preserves the topology and properties of the original cone. As shown above, the infinitesimal change $\delta \mathbf{v}_{\mathrm{c}}$ due to the contact forces lies in the contact space, which is parametrized by the contact velocity components $\mathbf{u}_{\mathrm{c}}$. From equation (15), the contact force components can be related to the infinitesimal change in the contact velocities as

$$
\lambda_{\mathrm{c}} \mathrm{d} t=\mathbf{M}_{\mathrm{c}} \delta \mathbf{u}_{\mathrm{c}}
$$

where $\mathbf{M}_{\mathrm{c}}=\left(\mathbf{A} \mathbf{M}^{-1} \mathbf{A}^{\mathrm{T}}\right)^{-1}$ is the effective mass matrix of the system in the contact space. The elements of effective mass can be derived from the elements of the inverse effective mass

$$
\mathbf{M}_{\mathrm{c}}^{-1}=\mathbf{A} \mathbf{M}^{-1} \mathbf{A}^{\mathrm{T}}=\left[\begin{array}{lll}
\mathbf{A}_{\mathrm{t}} \mathbf{M}^{-1} \mathbf{A}_{\mathrm{t}}^{\mathrm{T}} & \mathbf{A}_{\mathrm{t}} \mathbf{M}^{-1} \mathbf{A}_{\mathrm{n}}^{\mathrm{T}} \\
\mathbf{A}_{\mathrm{n}} \mathbf{M}^{-1} \mathbf{A}_{\mathrm{t}}^{\mathrm{T}} & \mathbf{A}_{\mathrm{n}} \mathbf{M}^{-1} \mathbf{A}_{\mathrm{n}}^{\mathrm{T}}
\end{array}\right]=\left[\begin{array}{cc}
\mathbf{H} & \mathbf{h} \\
\mathbf{h}^{\mathrm{T}} & a
\end{array}\right]
$$


Here, the $2 \times 2$ matrix $\mathbf{H}=\mathbf{A}_{\mathrm{t}} \mathbf{M}^{-1} \mathbf{A}_{\mathrm{t}}^{\mathrm{T}}$ and the scalar $a=\mathbf{A}_{\mathrm{n}} \mathbf{M}^{-1} \mathbf{A}_{\mathrm{n}}^{\mathrm{T}}$ account for the mass along the tangent and normal directions, respectively. The off-diagonal element $\mathbf{h}=\mathbf{A}_{\mathrm{t}} \mathbf{M}^{-1} \mathbf{A}_{\mathrm{n}}^{\mathrm{T}}$ reflects the coupling between the normal and tangential directions, which will be of interest later.

Then, the elements of the mass matrix

$$
\mathbf{M}_{\mathrm{c}}=\left[\begin{array}{cc}
\mathbf{M}_{\mathrm{t}} & \mathbf{m}_{\mathrm{tn}} \\
\mathbf{m}_{\mathrm{tn}}^{\mathrm{T}} & m_{\mathrm{n}}
\end{array}\right]
$$

can be computed from Eq. (25) by using the block matrix invesion formula as [36]

$$
\mathbf{M}_{\mathrm{c}}=\left[\begin{array}{cc}
\mathbf{H} & \mathbf{h} \\
\mathbf{h}^{\mathrm{T}} & a
\end{array}\right]^{-1}=\left[\begin{array}{cc}
\left(\mathbf{H}-\mathbf{h} a^{-1} \mathbf{h}^{\mathrm{T}}\right)^{-1} & \mathbf{m}_{\mathrm{tn}} \\
\mathbf{m}_{\mathrm{tn}}^{\mathrm{T}} & \left(a-\mathbf{h}^{\mathrm{T}} \mathbf{H}^{-1} \mathbf{h}\right)^{-1}
\end{array}\right]
$$

where the off diagonal term $\mathbf{m}_{\mathrm{tn}}=-\mathbf{H}^{-1} \mathbf{h} m_{\mathrm{n}}$ can also be written as $\mathbf{m}_{\mathrm{tn}}=-\mathbf{M}_{\mathrm{t}} \mathbf{h} a^{-1}$. Moreover, the fist diagonal term $\mathbf{M}_{\mathrm{t}}=\left(\mathbf{H}-\mathbf{h} a^{-1} \mathbf{h}^{\mathrm{T}}\right)^{-1}$ can be interpreted as the effective mas matrix associated with dynamics of the tangential velocity of a sliding contact, if the normal velocity is constrained.

Then, the quadratic expression for the projection of the generalized friction cone $\kappa_{\mathrm{g}}$ in terms of the contact velocity changes $\delta \mathbf{u}_{\mathrm{c}}$ can be obtained by applying the variable transformation in (24) to (21), which yields

$$
\kappa_{\mathrm{g}}\left(\delta \mathbf{u}_{\mathrm{c}}\right)=\delta \mathbf{u}_{\mathrm{c}}^{\mathrm{T}} \mathbf{Q} \delta \mathbf{u}_{\mathrm{c}}=\left[\begin{array}{l}
\delta \mathbf{u}_{\mathrm{t}} \\
\delta u_{\mathrm{n}}
\end{array}\right]^{\mathrm{T}}\left[\begin{array}{cc}
\mathbf{Q}_{\mathrm{t}} & \mathbf{Q}_{\mathrm{tn}} \\
\mathbf{Q}_{\mathrm{tn}}^{\mathrm{T}} & Q_{\mathrm{n}}
\end{array}\right]\left[\begin{array}{l}
\delta \mathbf{u}_{\mathrm{t}} \\
\delta u_{\mathrm{n}}
\end{array}\right]=0
$$

where $\mathbf{Q}=\mathbf{M}_{\mathrm{c}} \mathbf{Q}_{\mu} \mathbf{M}_{\mathrm{c}}$ is the matrix of the quadratic equation of the cone, and its elements are

$$
\begin{aligned}
\mathbf{Q}_{\mathrm{t}} & =\mathbf{M}_{\mathrm{t}}\left(\mathbf{I}_{2}-\frac{\mu^{2}}{a^{2}} \mathbf{h}^{\mathrm{T}}\right) \mathbf{M}_{\mathrm{t}} \\
Q_{\mathrm{n}} & =\left(\mathbf{h}^{\mathrm{T}} \mathbf{H}^{-2} \mathbf{h}-\mu^{2}\right) m_{\mathrm{n}}^{2} \\
\mathbf{Q}_{\mathrm{tn}} & =\left(\mathbf{M}_{\mathrm{t}}-\mu^{2} m_{\mathrm{n}} \mathbf{I}\right) \mathbf{m}_{\mathrm{tn}}
\end{aligned}
$$

where $\mathbf{I}_{2}$ is the $2 \times 2$ identity matrix. The conic surface $\kappa_{\mathrm{g}}$ in Eq. (28) lives in a 3-dimensional space, regardless of the dimension of the velocity space $n$.

As in the classic cone $\kappa_{\mu}$, the friction coefficient affects the geometry of the generalized cone $\kappa_{\mathrm{g}}$. For $\mu=0$ the cone degenerates into a line given by the parametrization

$$
\delta \mathbf{u}_{\mathrm{c}}\left(\lambda_{\mathrm{n}}\right)=\mathbf{M}_{\mathrm{c}}^{-1}\left[\begin{array}{c}
\mathbf{0} \\
\lambda_{\mathrm{n}}
\end{array}\right] \mathrm{d} t=\mathbf{A} \mathbf{M}^{-1} \mathbf{A}_{\mathrm{n}}^{\mathrm{T}} \lambda_{\mathrm{n}} \mathrm{d} t
$$

which represents the space of constrained motion [36] associated with the unilateral contact constraint and projected onto the contact space, also known as natural contact direction in [37]. It can be interpreted as the direction in which the contact velocity changes due to the normal contact force alone. In case of frictionless collisions, this direction is important because all non-impulsive forces are usually neglected and only the impulses of the normal force are taken into account, and so the contact velocity only changes along this direction. On the other hand, for $\mu \rightarrow \infty$ the cone degenerates into a plane given by the parametrization

$$
\delta \mathbf{u}_{\mathrm{c}}\left(\boldsymbol{\lambda}_{\mathrm{t}}\right)=\mathbf{M}_{\mathrm{c}}^{-1}\left[\begin{array}{c}
\boldsymbol{\lambda}_{\mathrm{t}} \\
0
\end{array}\right] \mathrm{d} t=\mathbf{A} \mathbf{M}^{-1} \mathbf{A}_{\mathrm{t}}^{\mathrm{T}} \boldsymbol{\lambda}_{\mathrm{t}} \mathrm{d} t
$$

which contains the directions in which the contact velocity can change due to the friction force. Note that this plane is not directly related to either the tangent plane or the natural contact direction.

Figure 2 shows the proposed generalized friction cone for the so-called Painlevé example of a single rod in contact with a plane. ${ }^{1}$ The shape of the cone is fully determined by the friction coefficient $\mu$ and the orientation of the rod with respect to the plane $\left(\theta=45^{\circ}\right.$ in this case), since the rod is assumed to have a homogeneous mass distribution. This is because the equation of the cone (28) only depends on the configuration and mass distribution of the system.

\footnotetext{
${ }^{1}$ Interestingly, the original example used by Painlevé in [2] consists of a cylinder with one of its bases in contact with a slope, which is sliding down the slope with a velocity parallel to the surface. Although the mass distribution of the cylinder is different from the one of a rod, the same conclusions can be drown assuming one single contact point at the edge of the cylinder base.
} 

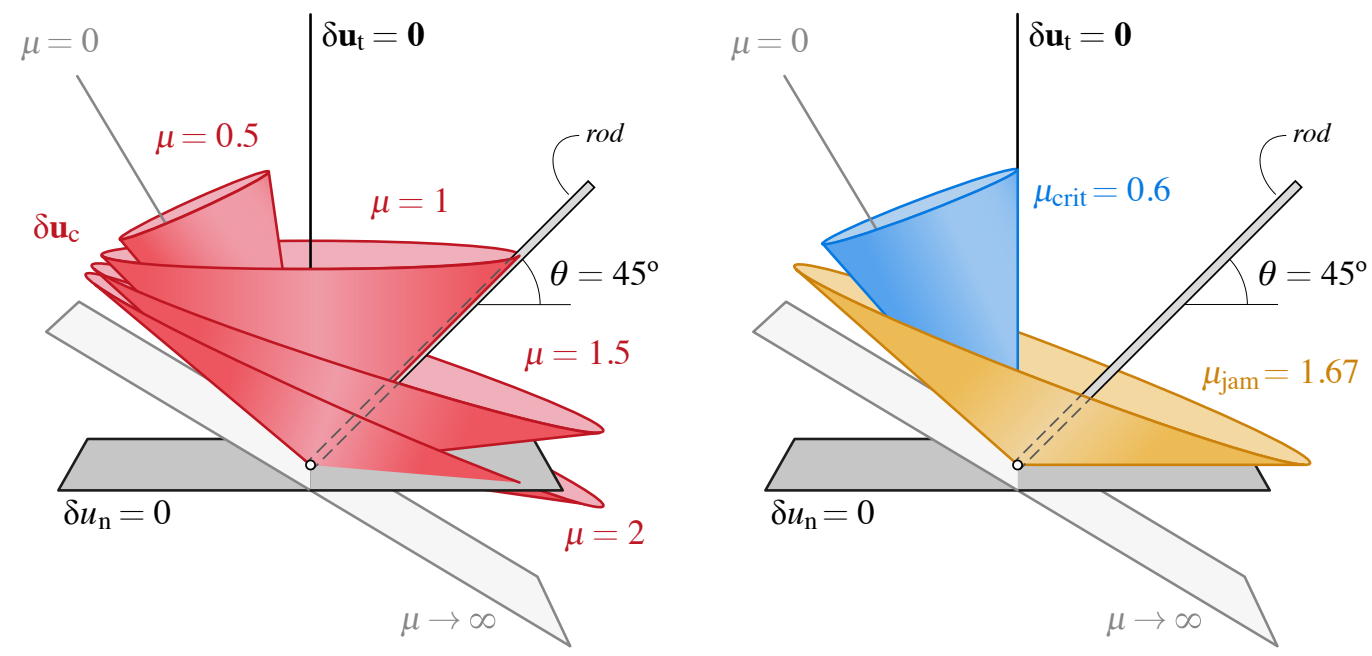

Fig. 2 The generalized friction cone of a rod in contact with the ground at $\theta=45^{\circ}$ for different values of $\mu$ (left), and for the critical and jamming friction coefficients $\mu_{\text {crit }}$ and $\mu_{\text {jam }}$.

In general, $\mathbf{Q}$ is a full-rank symmetric matrix that represents an elliptic cone $\kappa_{\mathrm{g}}$ without any particular shape. Nevertheless, its geometry in some cases can be of interest and help to better understand the dynamics of multibody systems with frictional contacts. For instance, it is clear from (28) that the direction $\delta \mathbf{u}_{\mathrm{t}}=\mathbf{0}$ is located inside the cone if and only if $Q_{\mathrm{n}} \leqslant 0$, which, according to (30), it happens when the friction coefficient is greater than a critical value

$$
\mu \geqslant \mu_{\text {crit }}=\left\|\mathbf{H}^{-1} \mathbf{h}\right\|=\sqrt{\mathbf{A}_{\mathrm{n}} \mathbf{M}^{-1} \mathbf{A}_{\mathrm{t}}^{\mathrm{T}}\left(\mathbf{A}_{\mathrm{t}} \mathbf{M}^{-1} \mathbf{A}_{\mathrm{t}}^{\mathrm{T}}\right)^{-2} \mathbf{A}_{\mathrm{t}} \mathbf{M}^{-1} \mathbf{A}_{\mathrm{n}}^{\mathrm{T}}}
$$

where $\mu_{\text {crit }}$ is the critical friction coefficient and depends on the configuration and mass distribution of the system. Additionally, it plays an important role in single-point collisions with friction, in which sliding cannot restart if $\mu \geqslant \mu_{\text {crit }}[24,38]$. This fact is consistent with the generalized cone proposed here, because in such a case, static friction is able to develop a contact force that generates $\delta \mathbf{u}_{\mathrm{t}}=\mathbf{0}$, i.e., a force inside the cone (see Fig. 2).

Another interesting aspect of the generalized cone is its intersection with the plane $\delta u_{\mathrm{n}}=0$, a degenerate conic described by the quadratic equation

$$
\delta \mathbf{u}_{\mathrm{t}}^{\mathrm{T}} \mathbf{Q}_{\mathrm{t}} \delta \mathbf{u}_{\mathrm{t}}=0
$$

which represents a point, the cone vertex, if $\mathbf{Q}_{\mathrm{t}}$ is positive definite (for small $\mu$ ), or two lines that intersect at the cone vertex if $\mathbf{Q}_{\mathrm{t}}$ is indefinite (for large $\mu$ ). The threshold value makes $\mathbf{Q}_{\mathrm{t}}$ be positive semi-definite (i.e., $\operatorname{det} \mathbf{Q}_{\mathrm{t}}=0$ ) and, according to (29), this happens when $\mathbf{h}^{\mathrm{T}} \mathbf{h}=a^{2} / \mu^{2}$, which is the non-zero eigenvalue of the rank-1 matrix $\mathbf{h h}^{\mathrm{T}}$. Therefore, the intersection is different from a point if

$$
\mu \geqslant \mu_{\mathrm{jam}}=\frac{a}{\|\mathbf{h}\|}=\frac{\mathbf{A}_{\mathrm{n}} \mathbf{M}^{-1} \mathbf{A}_{\mathrm{n}}^{\mathrm{T}}}{\sqrt{\mathbf{A}_{\mathrm{n}} \mathbf{M}^{-1} \mathbf{A}_{\mathrm{t}}^{\mathrm{T}} \mathbf{A}_{\mathrm{t}} \mathbf{M}^{-1} \mathbf{A}_{\mathrm{n}}^{\mathrm{T}}}}
$$

where $\mu_{\mathrm{jam}}$ is the jamming friction coefficient, which depends on the configuration and mass distribution of the system [26] (see Fig. 2). If the generalized friction cone dips below the tangent plane, certain contact forces can generate a negative change in the contact normal velocity, i.e., $\delta u_{\mathrm{n}}<0$. This can cause dynamic locking [9,23,24], which gives rise to the Painlevé-like situations $[9,16,28]$, where the dynamic equations of a rigid body with Coulomb friction have no solution or even several possible solutions.

Similarly, if multiple contact points are present in the system, the equation of the generalized friction cone can still be derived for each contact point. If constraints are used to model contact, the rest of contacts would affect the dynamics of the contact point of interest. Therefore, the effective mass at the contact point under study in Eq. (24) would be different, which would make the shape of the generalized friction cone change as well. Although we focus our study on multibody systems with one single contact point, it can still give some insight into the contact problem and friction coupling in more complex systems. 


\section{Solvability of Dynamic Formulations}

Solvability of the dynamic formulation can be compromised in presence of Coulomb friction, as first showed by Painlevé [2]. Even for a system of one body sliding on a fixed surface, it can be shown that the dynamic equations may have several solutions or none [28]. In this section, and following a general approach, different scenarios where the Coulomb friction can lead to paradoxical situations are analyzed using the proposed representation of the generalized friction cone.

According to the Coulomb model (20), the direction of the kinetic friction force is determined by the sliding direction $\mathbf{e}_{t}$, and so the contact force can be written as

$$
\lambda_{\mathrm{c}}=\left[\begin{array}{c}
-\mu_{\mathrm{k}} \mathbf{e}_{\mathrm{t}} \\
1
\end{array}\right] \lambda_{\mathrm{n}}
$$

which depends on the normal force component $\lambda_{\mathrm{n}}$ and lies on the friction cone $\kappa_{\mu}$. The problem arises when the contact force $\boldsymbol{\lambda}_{\mathrm{c}}$ would contribute to a negative change in the normal velocity component, i.e, $\delta u_{\mathrm{n}}<0$, which may happen if part of the generalized friction cone is found below the tangent plane [28]. The change in the normal velocity component is given as function of the normal contact force $\lambda_{\mathrm{n}}$ as

$$
\delta u_{\mathrm{n}}=\mathbf{A}_{\mathrm{n}} \mathbf{M}^{-1} \mathbf{A}^{\mathrm{T}} \boldsymbol{\lambda}_{\mathrm{c}} \mathrm{d} t=\left(a-\mu_{\mathrm{k}} \mathbf{h}^{\mathrm{T}} \mathbf{e}_{\mathrm{t}}\right) \lambda_{\mathrm{n}} \mathrm{d} t
$$

and it can be negative only if sliding happens in a direction $\mathbf{e}_{t}$ so that

$$
\mathbf{e}_{\mathrm{jam}}^{\mathrm{T}} \mathbf{e}_{\mathrm{t}}>\frac{\mu_{\mathrm{jam}}}{\mu_{\mathrm{k}}}
$$

where

$$
\mathbf{e}_{\mathrm{jam}}=\frac{\mathbf{h}}{\|\mathbf{h}\|}=\frac{\mathbf{A}_{\mathrm{t}} \mathbf{M}^{-1} \mathbf{A}_{\mathrm{n}}^{\mathrm{T}}}{\sqrt{\mathbf{A}_{\mathrm{n}} \mathbf{M}^{-1} \mathbf{A}_{\mathrm{t}}^{\mathrm{T}} \mathbf{A}_{\mathrm{t}} \mathbf{M}^{-1} \mathbf{A}_{\mathrm{n}}^{\mathrm{T}}}}
$$

which defines the direction in the tangent plane coupled with the normal component.

If the sliding velocity is such that the condition in Eq. (39) is satisfied, the normal force contributes to a negative change in the normal velocity, which causes dynamic jamming [26, 27, 28]. The higher the coupling between normal and tangential components, the more likely it is to occur. According to Eq. (36), the jamming friction coefficient $\mu_{\mathrm{jam}}$ becomes low when the coupling is high, and it tends to infinity when the normal and tangential components are completely decoupled, i.e., $\mathbf{h}=\mathbf{0}$. Moreover, the sliding direction defined by $\mathbf{e}_{\text {jam }}$ results in the most negative change of the normal velocity, which makes it the sliding direction where jamming is more likely to happen.

Dynamic jamming may occur if sliding happens in a certain direction, and only if $\mu_{\mathrm{k}}>\mu_{\mathrm{jam}}$. All these sliding directions define a continuous region in the tangent plane defined by the condition in Eq. (39). Therefore, we can define a locking angle $\alpha$ that quantifies the aperture angle of this region. Given that $\mathbf{e}_{j a m}^{\mathrm{T}} \mathbf{e}_{\mathrm{t}}=\cos \beta \in[-1,+1]$, where $\beta$ is the angle between the sliding direction and the coupled direction $\mathbf{e}_{\mathrm{jam}}$, the locking angle can be defined as

$$
\alpha=2 \arccos \left(\frac{\mu_{\mathrm{jam}}}{\mu_{\mathrm{k}}}\right)
$$

which only exists if $\mu_{\mathrm{k}}>\mu_{\mathrm{jam}}$. The maximum value for the locking angle is $\alpha=180^{\circ}$ (i.e., half the plane), which happens when the jamming friction coefficient becomes zero. This value can never be reached in reality, but $\mu_{\mathrm{jam}}$ can become small when the coupling between normal and tangential directions is high.

Figure 3 shows the locking angle $\alpha$ for a single rod contacting on the ground at different angles $\theta$ and different values of the friction coefficient $\mu_{\mathrm{k}}$. As it can be seen, the locking angle only exists (i.e., $\alpha>0$ ) if the friction coefficient $\mu_{\mathrm{k}}>\frac{4}{3}[15,28]$. A top view of the generalized cone for a rod at $45^{\circ}$ is shown on the right of Fig. 3 and, in this case, the locking angle is $\alpha=67.1^{\circ}$. As discussed above, all the sliding directions in which jamming occurs generate $\delta u_{\mathrm{n}}<0$, and so this is reflected on the part of the cone that dips below the tangent plane. Note that the aperture of the intersection of the cone with the plane is not equal to the locking angle. This is because the locking angle $\alpha$ is associated with the sliding direction defined by the sliding velocity $\mathbf{u}_{\mathrm{t}}$, whereas the generalized friction cone is associated with the change in contact velocity $\delta \mathbf{u}_{\mathrm{c}}$. Nevertheless, the cone can only intersect with the plane if $\mu_{\mathrm{k}}>\mu_{\mathrm{jam}}$, and in such a case the locking angle $\alpha>0$, and so dynamic jamming may occur. 
Let us consider the single-rod example in contact with a plane at $\theta=45^{\circ}$ in order to assess and discuss the solvability of dynamic formulations by using the generalized friction cone. Figure 4 illustrates different possible situations in which the solution may be compromised. For relatively small friction coefficients $\left(\mu<\mu_{\text {jam }}\right)$, the generalized cone is completely above the tangent plane, and so all possible contact forces contribute to a positive change in the normal velocity component, i.e., $\delta u_{\mathrm{n}}>0$. In such a case, the contact detaches if the contribution of the other forces to the change in contact velocity $\left(\delta \mathbf{u}_{0}\right)$ pulls the contact apart (Fig. 4 , top). Whereas if the contribution of the other forces pushes it together (Fig. 4, bottom), the contribution of the contact force to the change in contact velocity $\delta \mathbf{u}_{\mathrm{c}}$ is able to balance the total change in velocity d $\mathbf{u}_{\mathrm{c}}=\delta \mathbf{u}_{\mathrm{c}}+\delta \mathbf{u}_{0}$ and the contact remains closed, i.e., $\mathrm{d} u_{\mathrm{n}}=0$. Therefore, a unilateral constraint can be used to represent the normal contact force in such cases, because the constraint equation $\dot{u}_{\mathrm{n}} \geqslant 0$ can be satisfied.

On the other hand, if the friction coefficient is high enough $\left(\mu>\mu_{\mathrm{jam}}\right)$, part of the cone dips below the tangent plane, and some contact forces can contribute to a negative change in the normal velocity component, i.e., $\delta u_{\mathrm{n}}<0$. Then, if the contribution of the other forces to the change in the contact velocity $\left(\delta \mathbf{u}_{0}\right)$ pushes the contact together, the total change in the velocity $\mathrm{d} \mathbf{u}_{\mathrm{c}}=\delta \mathbf{u}_{\mathrm{c}}+\delta \mathbf{u}_{0}$ has a negative normal component, i.e., $\mathrm{d} u_{\mathrm{n}}<0$ (Fig. $4 . \mathrm{b}$, bottom). Dynamic jamming occurs when the contact slides in a direction such that the jamming condition in Eq. (39) is satisfied, and so the contact force contributes to a negative change in the normal velocity. In such a case, the contact enters in a compression phase where the normal velocity decreases $\left(u_{\mathrm{n}}<0\right)$ and the contact interface starts compressing. The normal velocity keeps decreasing until the contact stops sliding, instant at which the normal
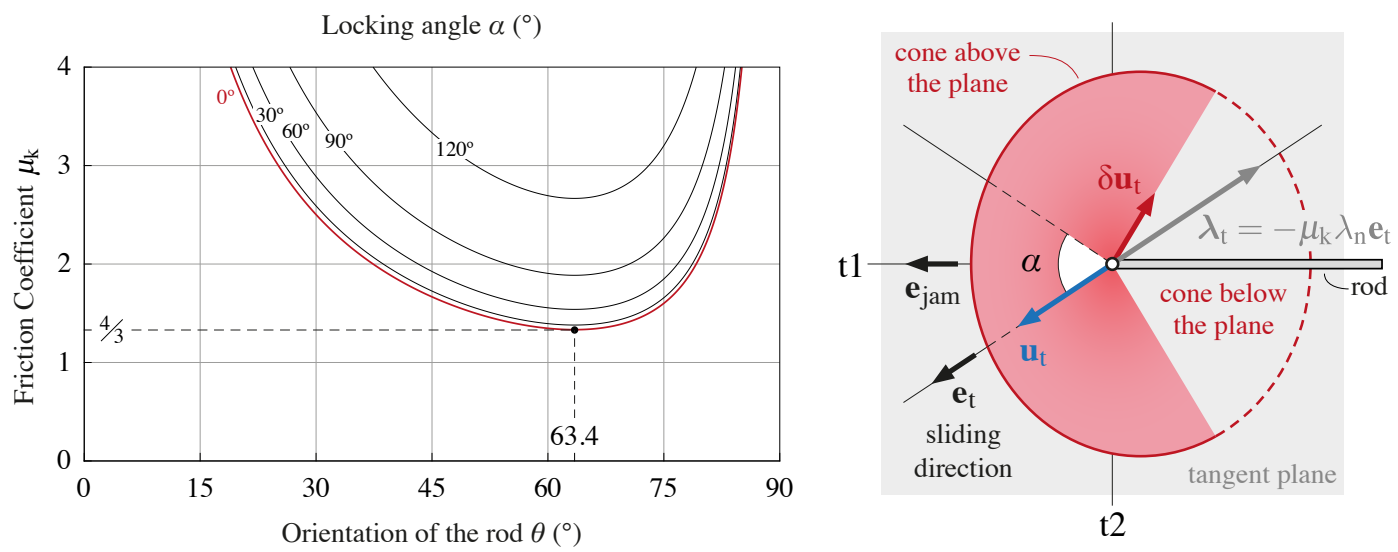

Fig. 3 Range of sliding velocities $\mathbf{u}_{\mathrm{t}}$ that contribute to a negative change of the normal contact velocity $\delta u_{\mathrm{n}}$ for a single rod in contact with the ground at $\theta=45^{\circ}$ and $\mu=2$, with locking angle $\alpha=67,1^{\circ}$ (right), and value of friction coefficient $\mu$ in terms of the orientation of the rod $\theta$, for different values of locking angle $\alpha$ (left).

(a) Unique Solutions $(\mu=1)$

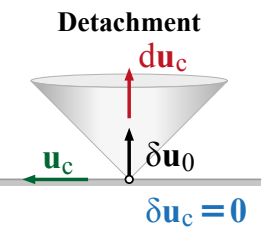

Sliding Contact

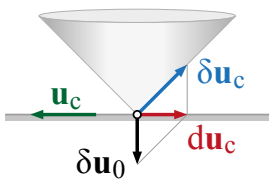

(b) Realistic Solutions $(\mu=2)$

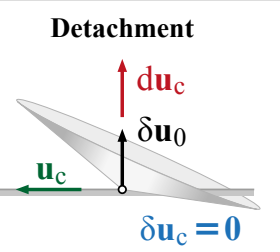

Dynamic Jamming

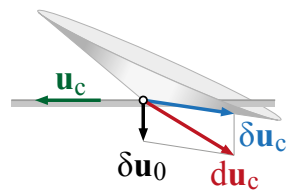

(c) Unrealistic Solutions $(\mu=2)$

Cohesion

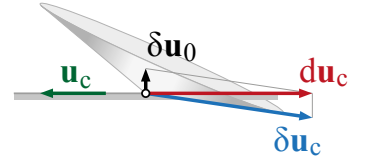

Negative Dissipation

Fig. 4 Possible solutions of a sliding rod at $\theta=45^{\circ}$ with a plane for $\mu=1$ (a), and $\mu=2$ (b, c); where $\mu_{\text {jam }}=1.67$ in this configuration. The other external forces $\delta \mathbf{u}_{0}$ can push the contact together (top), and pull it apart (bottom). 
velocity is still negative. After the sliding phase, the compression phase continues but without sliding $\left(\mathbf{u}_{\mathrm{t}}=\mathbf{0}\right)$ until the normal velocity becomes zero $\left(u_{\mathrm{n}}=0\right)$ and the compression phase finally ends. Part of the energy of the system is dissipated during the compression phase, and the rest is restored back to the system during the restitution phase, at the end of which the contact detaches with a positive normal velocity. This phenomenon is characterized by a rapid increase in the contact forces, which tend to large values in a short period of time and generate a sudden change in the velocities, like an impact without collision [24,25].

To model this effect, and be able to solve the dynamic equation, it is possible to use formulations based on contact forces defined via constitutive laws [30], or formulations at the impulse-momentum level [29]. However, if a unilateral constraint $\dot{u}_{\mathrm{n}} \geqslant 0$ is used, according to (37), the normal force would need to be negative $\left(\lambda_{\mathrm{n}}<0\right)$ to be able to generate $\delta u_{\mathrm{n}} \geqslant 0$ and keep the constraint (Fig. 4.c, top). This unrealistic solution results in a contact force that develops negative dissipation, and so energy would be generated due to friction. In this case, the dynamics formulation leads to a linear complementarity problem (LCP) that has no solution, as shown in the next section.

Finally, in case the other forces contribute to a change in the contact velocity $\left(\delta \mathbf{u}_{0}\right)$ such that the contact is pulled apart, detachment should occur (Fig. 4.b, top). However, the contact force contributes to a negative change in the normal velocity, i.e., $\delta u_{\mathrm{n}}<0$, it can balance the other forces and can keep the body in contact with $\mathrm{d} u_{\mathrm{n}}=0$ (Fig. 4.c, top). This unrealistic solution gives a false effect of cohesion, because the contact should detach, but the contact force prevents it. To solve this paradoxical situation, Painlevé [3] proposed a principle that prevents friction from keeping the contact closed. ${ }^{2}$ Essentially, it states that two bodies should not interact (i.e., the contact should detach), if they would not interact under the same conditions but in the absence of friction $(\mu=0)$. Unfortunately, this principle is only applicable to individual contact pairs and cannot be generalized to the multiple-point case, where friction in the system can prevent some contact pairs from detaching.

\section{Example of a System with Single Contact}

Cases where the dynamic equations may become ill-posed not only occur for very high values of the friction coefficient, which can be considered unrealistic, as in the Painlevé paradox. In this section, an example consisting of an articulated arm in contact with a plane is used to show that it is possible to formulate the dynamic equations of a system with realistic parameters and have no solution. Additionally, the generalized friction cone proposed in this paper helps to determine the cases where the solution is compromised.

The articulated arm in Fig. 5 consists of 4 bodies connected by revolute joints and it is in contact with the fixed horizontal plane at point $\mathrm{P}$. The configuration space can be defined by the joint angles $\mathbf{q}=\left[\theta_{1}, \theta_{2}, \theta_{3}, \varphi\right]^{\mathrm{T}}$,
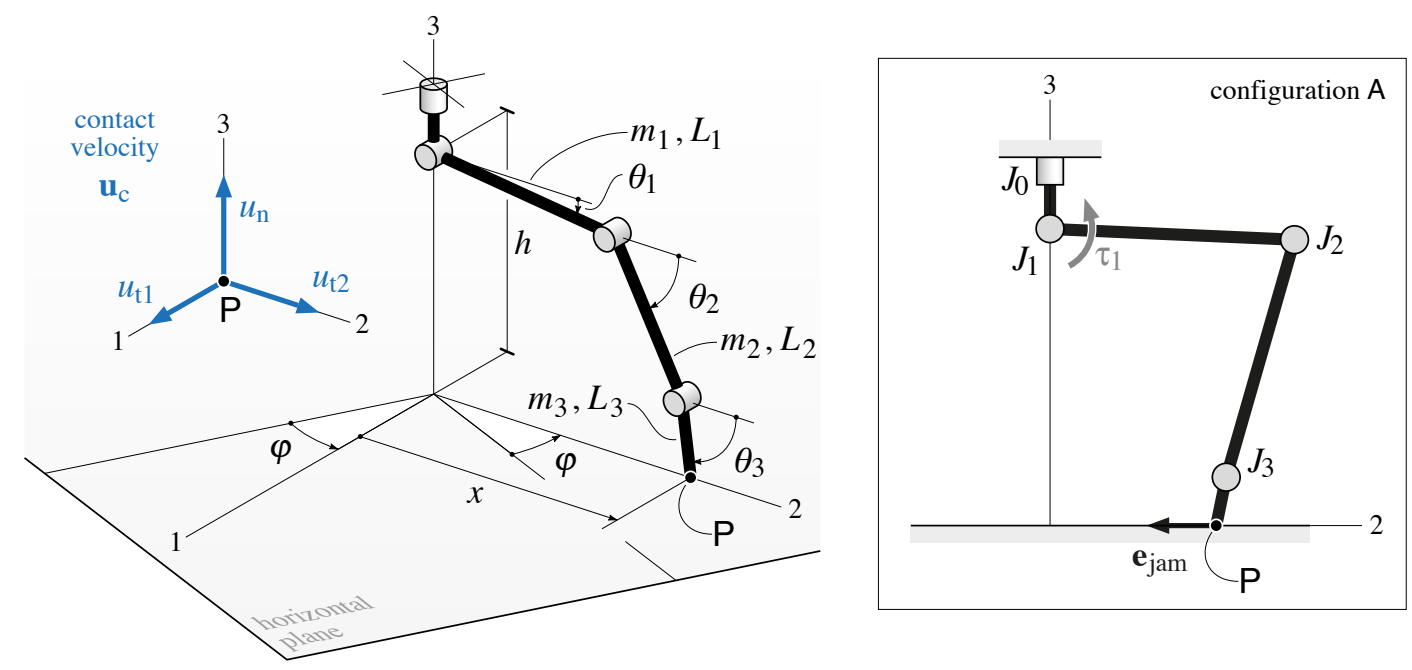

Fig. 5 Articulated arm in contact with a plane (left) and the configuration A that corresponds to a local minimum of the friction coefficient $\mu_{\text {jam }}$ (right). The system dimensions are $L_{1}=L_{2}=0.5 \mathrm{~m}, L_{3}=0.1 \mathrm{~m}$, and $h=0.6 \mathrm{~m}$, with a uniform linear mass density $\rho=2 \mathrm{~kg} / \mathrm{m}$.

\footnotetext{
${ }^{2}$ The original text in french by Painlevé [3] can be translated as [28]: "Two rigid bodies, which under given conditions would not produce any pressure on one another if they were ideally smooth, would likewise not act on one another if they were rough."
} 
and its time derivatives can be used as generalized velocities $\mathbf{v}=\dot{\mathbf{q}}$, i.e., $n=p=4$. Therefore, the mass matrix associated with this parametrization is

$$
\mathbf{M}=\left[\begin{array}{cccc}
\frac{\rho L_{1}^{2}}{3}\left(L_{1}+3 L_{2}+3 L_{3}\right) & \frac{\rho L_{1} L_{2}}{2}\left(L_{2}+2 L_{3}\right) \cos \left(\theta_{1}-\theta_{2}\right) & \frac{\rho L_{1} L_{3}^{2}}{2} \cos \left(\theta_{1}-\theta_{3}\right) & 0 \\
\frac{\rho L_{1} L_{2}}{2}\left(L_{2}+2 L_{3}\right) \cos \left(\theta_{1}-\theta_{2}\right) & \frac{\rho L_{2}^{2}}{3}\left(L_{2}+3 L_{3}\right) & \frac{\rho L_{2} L_{3}^{2}}{2} \cos \left(\theta_{2}-\theta_{3}\right) & 0 \\
\frac{\rho L_{1} L_{3}^{2}}{2} \cos \left(\theta_{1}-\theta_{3}\right) & \frac{\rho L_{2} L_{3}^{2}}{2} \cos \left(\theta_{2}-\theta_{3}\right) & \frac{\rho L_{3}^{3}}{3} & 0 \\
0 & 0 & 0 & I_{\varphi}
\end{array}\right]
$$

where $\rho$ is the linear mass density of the arm, which is considered the same for all the bodies, and $L_{1}, L_{2}$, and $L_{3}$ are the dimensions of the system (see, Fig. 5). The diagonal element of the mass matrix $I_{\varphi}$ represents the inertia of the arm associated with the coordinate $\varphi$, that is

$$
\begin{aligned}
I_{\varphi}= & \frac{\rho L_{1}^{3}}{3} \cos ^{2} \theta_{1}+\frac{\rho L_{2}^{3}}{12} \cos ^{2} \theta_{2}+\rho L_{2}\left(L_{1} \cos \theta_{1}+\frac{L_{2}}{2} \cos \theta_{2}\right)^{2} \\
& +\frac{\rho L_{3}^{3}}{12} \cos ^{2} \theta_{3}+\rho L_{3}\left(L_{1} \cos \theta_{1}+L_{2} \cos \theta_{2}+\frac{L_{3}}{2} \cos \theta_{3}\right)^{2}
\end{aligned}
$$

The three components $(r=3)$ of the contact velocity at $\mathrm{P}$ can be written as

$$
\mathbf{u}_{\mathrm{c}}=\left[\begin{array}{c}
u_{\mathrm{t} 1} \\
u_{\mathrm{t} 2} \\
\hline u_{\mathrm{n}}
\end{array}\right]=\left[\begin{array}{cccc}
0 & 0 & 0 & -x \\
-L_{1} \sin \theta_{1} & -L_{2} \sin \theta_{2} & -L_{3} \sin \theta_{3} & 0 \\
\hline-L_{1} \cos \theta_{1} & -L_{2} \cos \theta_{2} & -L_{3} \cos \theta_{3} & 0
\end{array}\right]\left[\begin{array}{c}
\dot{\theta}_{1} \\
\dot{\theta}_{2} \\
\dot{\theta}_{3} \\
\dot{\varphi}
\end{array}\right]=\left[\begin{array}{c}
\mathbf{A}_{\mathrm{t}} \\
\mathbf{A}_{\mathrm{n}}
\end{array}\right] \mathbf{v}
$$

where the coordinate $x=L_{1} \cos \theta_{1}+L_{2} \cos \theta_{2}+L_{3} \cos \theta_{3}$ is used to simplify the expressions, and defines the distance between point $P$ and the axis of the vertical revolute joint represented by $\varphi$ (see Fig. 5). Additionally, if the constraint $u_{\mathrm{n}}=0$ is considered while point $\mathrm{P}$ is in contact, the independent set of coordinates $\mathbf{s}=\left[x, \theta_{3}, \varphi\right]^{\mathrm{T}}$ can also be used.

To determine when the formulation can be solved, the limit values of the friction coefficient determined by the generalized friction cone will be used. The value of the friction coefficient $\mu_{\text {jam }}$ for different configurations of the arm are shown in Fig. 6, which only depends on the coordinates $x$ and $\theta_{3}$. The system shows two local minima, $\mu_{\mathrm{jam}}^{\mathrm{A}}=0.421$ and $\mu_{\mathrm{jam}}^{\mathrm{B}}=0.404$, which are fairly realistic friction coefficients.

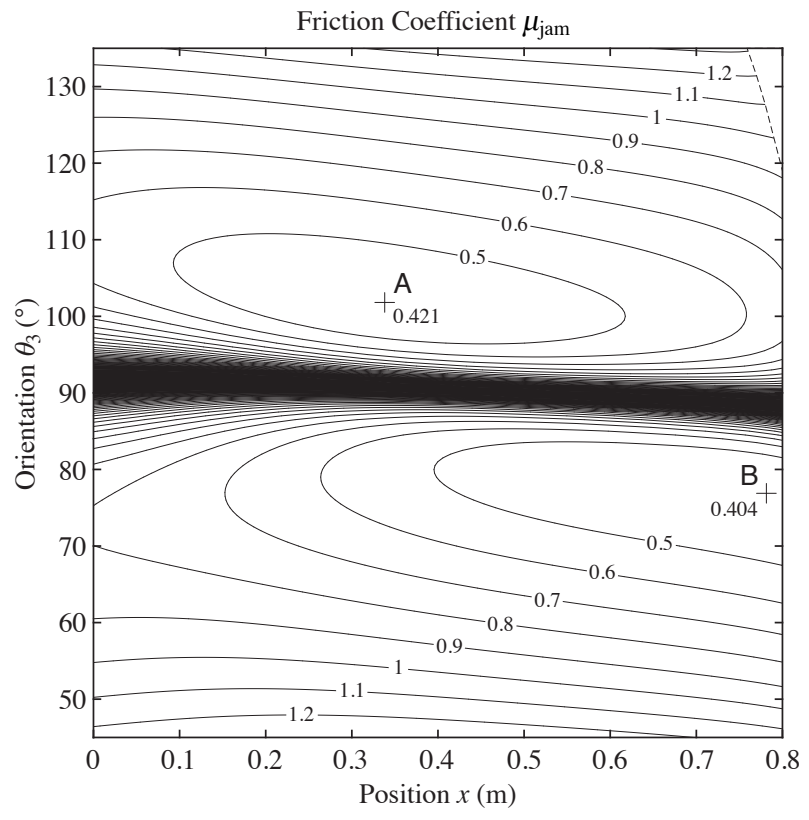

Fig. 6 Friction coefficient $\mu_{\mathrm{jam}}$ for the configuration space of the articulated arm in contact with a plane. 
Here we analyze the local minimum $A$, where system configuration $\mathbf{q}^{\mathrm{A}}=\left[2.5^{\circ}, 106.2^{\circ}, 101.8^{\circ}, \varphi\right]^{\mathrm{T}}$ is independent of $\varphi$ and corresponds to $x^{\mathrm{A}}=0.340 \mathrm{~m}$. If the system is in this configuration and the kinetic friction coefficient is large enough $\left(\mu_{\mathrm{k}}>\mu_{\mathrm{jam}}\right)$, the contact force may affect the contact velocity with $\delta u_{\mathrm{n}}<0$, which can lead to unrealistic solutions. For instance, if we take a value of the friction coefficient larger that $\mu_{\text {jam }}^{\mathrm{A}}=0.421$, such as $\mu_{\mathrm{k}}=0.5$, the locking angle is $\alpha=65.3^{\circ}$ according to Eq. (41). In such a case, the direction in the tangent plane coupled with the normal force is $\mathbf{e}_{\mathrm{jam}}=[0,-1]^{\mathrm{T}}$ (see Fig. 5).

Let us consider the system at the aforementioned configuration $\mathbf{q}^{\mathrm{A}}$, with a velocity such that it satisfies the jamming condition in Eq. (39). If we choose the time derivatives of the independent set of coordinates $\mathbf{S}$ to be $\dot{\mathbf{s}}=\left[\dot{x}, \dot{\theta}_{3}, \dot{\varphi}\right]^{\mathrm{T}}=[-1 \mathrm{~m} / \mathrm{s}, 0,1 \mathrm{rad} / \mathrm{s}]^{\mathrm{T}}$, then, the contact point velocity is

$$
\mathbf{u}_{\mathrm{c}}=\left[\begin{array}{c}
u_{\mathrm{t} 1} \\
u_{\mathrm{t} 2} \\
u_{\mathrm{n}}
\end{array}\right]=\left[\begin{array}{c}
-x \dot{\varphi} \\
\dot{x} \\
0
\end{array}\right]=\left[\begin{array}{c}
-0.340 \\
-1.0 \\
0
\end{array}\right] \mathrm{m} / \mathrm{s}
$$

where the sliding direction $\mathbf{e}_{\mathrm{t}}=[-0.3219,-0.9468]^{\mathrm{T}}$ satisfies the locking condition in Eq. (39). Therefore, only considering the effect of gravity, by (15) and (16) the total change in the contact velocity is

$$
\mathrm{d} \mathbf{u}_{\mathrm{c}}=\delta \mathbf{u}_{\mathrm{c}}+\delta \mathbf{u}_{0}=\left[\begin{array}{c}
0.1265 \\
8.316 \\
-0.3118
\end{array}\right] \lambda_{\mathrm{n}} \mathrm{d} t+\left[\begin{array}{c}
1.0 \\
-0.0169 \\
-9.766
\end{array}\right] \mathrm{d} t
$$

which depends on the normal force $\lambda_{\mathrm{n}}$, and has $\mathrm{d} u_{\mathrm{n}}<0$ for all $\lambda_{\mathrm{n}} \geqslant 0$.

If a kinematic constraint is used to model the contact, the following LCP problem can be formulated while the contact is sliding

$$
\left.\begin{array}{c}
\dot{u}_{\mathrm{n}}=-0.3118 \lambda_{\mathrm{n}}-9.766 \\
\dot{u}_{\mathrm{n}} \geqslant 0, \quad \lambda_{\mathrm{n}} \geqslant 0, \quad \dot{u}_{\mathrm{n}} \lambda_{\mathrm{n}}=0
\end{array}\right\}
$$

for which no solution exists. However, if the contact force is defined via a constitutive relation, the dynamic equations can be integrated, and in such a case, the contact interface is compressed because of $\delta u_{\mathrm{n}}<0$, until the contact stops sliding. It is also possible to formulate the dynamic equations at the impulse-momentum level.

On the other hand, under the same conditions as described above, if now a torque $\tau_{1}=5 \mathrm{Nm}$ is applied at the joint $J_{1}$ in the negative direction of angle $\theta_{1}$, so that point $\mathrm{P}$ tends to detach (see Fig. 5). Then, the total change in the contact velocity is

$$
\mathrm{d} \mathbf{u}_{\mathrm{c}}=\delta \mathbf{u}_{\mathrm{c}}+\delta \mathbf{u}_{0}=\left[\begin{array}{c}
0.1265 \\
8.316 \\
-0.3118
\end{array}\right] \lambda_{\mathrm{n}} \mathrm{d} t+\left[\begin{array}{c}
1.0 \\
-1.262 \\
3.223
\end{array}\right] \mathrm{d} t
$$

which yields the following LCP problem

$$
\left.\begin{array}{c}
\dot{u}_{\mathrm{n}}=-0.3118 \lambda_{\mathrm{n}}+3.223 \\
\dot{u}_{\mathrm{n}} \geqslant 0, \quad \lambda_{\mathrm{n}} \geqslant 0, \quad \dot{u}_{\mathrm{n}} \lambda_{\mathrm{n}}=0
\end{array}\right\}
$$

which has two solutions: the expected solution with detachment $\left(\lambda_{\mathrm{n}}=0\right.$ and $\left.\dot{u}_{\mathrm{n}}=3.223 \mathrm{~m} / \mathrm{s}^{2}\right)$, and the unrealistic solution without detachment $\left(\lambda_{\mathrm{n}}=10.3 \mathrm{~N}\right.$ and $\left.\dot{u}_{\mathrm{n}}=0\right)$. Both are solutions of the mathematical problem. However, only one is consistent with the physical problem at hand, and so additional information has to be provided to the solver in order to determine which is the physically meaningful solution.

\section{Conclusions}

Dynamic formulations for multibody systems with contacts and Coulomb friction can have no solution, or several, depending on the assumptions made. Namely, using kinematic constraints to model the contact when dynamic jamming (or locking) occurs may lead to unrealistic solutions of the dynamic equations. This can only be overcome by either using constitutive relations, or formulating the dynamic equations at the impulse-momentum level and integrate them with respect to the impulses within the contact interval, as in a collision. By following a general 
approach, the formulation of a multibody system dynamics in the contact space parametrized by the contact velocities was obtained. This formulation allows to define the effect of the contact forces to the contact velocities, which helps to gain a deeper insight into the system dynamics in presence of friction.

The projection of the generalized friction cone to the contact space was successfully used to analyze friction coupling and the solvability of the system dynamic equations. The fact that this is a 3-dimensional cone (parametrized by the 3 velocity components of a single contact point) makes it easier to visualize than its $n$ dimensional counterpart. Moreover, the quadratic equation of the proposed friction cone representation has been derived and analyzed, which allows for determining the threshold value of the friction coefficient beyond which the dynamic formulation may become ill-posed. It is possible to show that the so-called Painlevé paradox is not an isolated phenomenon that only happens for very high unrealistic values of the friction coefficient. Additionally, it is shown how multibody systems with realistic parameters can also undergo similar situations due to friction coupling, in which the solvability of the dynamic formulation may be compromised if contact is modelled via unilateral constraints.

\section{References}

1. E. Berger, Friction modeling for dynamic system simulation, Applied Mechanics Reviews 55(6), 535 (2002)

2. P. Painlevé, Sur les lois du frottement de glissement, Comptes Rendus Hebdomadaires des Séances de l'Académie des Sciences 121,112 (1895)

3. P. Painlevé, Sur les lois du frottement de glissement, Comptes Rendus Hebdomadaires des Séances de l'Académie des Sciences 141, 401 (1905)

4. J. Moreau, Quadratic programming in mechanics: Dynamics of one sided constraints, SIAM Journal on Control 4(1), 153 (1966)

5. P. Lötstedt, Mechanical systems of rigid bodies subject to unilateral constraints, SIAM Journal of Applied Mathematics 42(2), 281 (1982)

6. C. Lemke, On complementary pivot theory, Mathematics of the decision sciences, Lectures in applied mathematics 2, 95 (1968)

7. J. Júdice, F. Pires, Basic-set algorithm for a generalized linear complementarity problem, Journal of optimization theory and applications 74(3), 391 (1992)

8. K. Murty, Linear Complementarity, Linear and Nonlinear Programming (Heldermann, Berlin, Germany, 1988)

9. B. Brogliato, Nonsmooth mechanics (Springer, 1999)

10. P.D. Panagiotopoulos, in Hemivariational Inequalities (Springer, 1993), pp. 99-134

11. C. Glocker, Set-Valued Force Laws (Springer, Troy, New York, USA, 2001)

12. D.E. Stewart, J.C. Trinkle, An implicit time-stepping scheme for rigid body dynamics with inelastic collisions and Coulomb friction, International Journal for Numerical Methods in Engineering 39, 2673 (1996)

13. M. Anitescu, F.A. Potra, Formulating dynamic multi-rigid-body contact problems with friction as solvable linear complementarity problems, Nonlinear Dynamics 14, 231 (1997)

14. P. Lötstedt, Coulomb friction in two-dimensional rigid body systems, ZAMM-Journal of Applied Mathematics and Mechanics/Zeitschrift für Angewandte Mathematik und Mechanik 61(12), 605 (1981)

15. F. Pfeiffer, C. Glocker, Multibody Dynamics with unilateral Contacts (Wiley, New York, 1996)

16. D.E. Stewart, Rigid-body dynamics with friction and impact, SIAM Review 42, 3 (2000)

17. V. Acary, F. Cadoux, C. Lemaréchal, J. Malick, A formulation of the linear discrete coulomb friction problem via convex optimization, ZAMM-Journal of Applied Mathematics and Mechanics/Zeitschrift für Angewandte Mathematik und Mechanik 91(2), 155 (2011)

18. D.M. Kaufman, S. Sueda, D.L. James, D.K. Pai, Staggered projections for frictional contact in multibody systems, ACM Transactions on Graphics (TOG) 27(5), 164 (2008)

19. M. Anitescu, A. Tasora, An iterative approach for cone complementarity problems for nonsmooth dynamics, Computational Optimization and Applications 47(2), 207 (2010)

20. G. De Saxcé, Z.Q. Feng, The bipotential method: a constructive approach to design the complete contact law with friction and improved numerical algorithms, Mathematical and computer modelling 28(4-8), 225 (1998)

21. J. Li, G. Daviet, R. Narain, F. Bertails-Descoubes, M. Overby, G.E. Brown, L. Boissieux, An implicit frictional contact solver for adaptive cloth simulation, ACM Transactions on Graphics (TOG) 37(4), 52 (2018)

22. A. Charles, P. Ballard, The formulation of dynamical contact problems with friction in the case of systems of rigid bodies and general discrete mechanical systems-Painlevé and kane paradoxes revisited, Zeitschrift für angewandte Mathematik und Physik 67(4), 99 (2016)

23. J.J. Moreau, in Nonsmooth Mechanics and Applications (CISM Courses \& Lectures) (Springer, 1988), pp. 1-82

24. J.A. Batlle, On Newton's and Poisson's rules of percussive dynamics, Journal of Applied Mechanics 60(2), 376 (1993)

25. Z. Zhao, C. Liu, W. Ma, B. Chen, Experimental investigation of the Painlevé paradox in a robotic system, Journal of Applied Mechanics 75(4), 041006 (2008)

26. J.A. Batlle, S. Cardona, The jamb (self-locking) process in three-dimensional collisions, Journal of Applied Mechanics 65(2), 417 (1998)

27. W.J. Stronge, in Proceedings of the Royal Society of London A: Mathematical, Physical and Engineering Sciences, vol. 431 (The Royal Society, 1990), vol. 431, pp. 169-181

28. F. Génot, B. Brogliato, New Results on Painlevé Paradoxes. Tech. Rep. RR-3366, INRIA (1998). URL https://hal.inria.fr/inria-00073323

29. D.E. Stewart, Convergence of a time-stepping scheme for rigid-body dynamics and resolution of Painlevé's problem, Archive for Rational Mechanics and Analysis 145(3), 215 (1998) 
30. Y. Shen, W. Stronge, Painlevé paradox during oblique impact with friction, European Journal of Mechanics-A/Solids 30(4), 457 (2011)

31. R.I. Leine, B. Brogliato, H. Nijmeijer, Periodic motion and bifurcations induced by the Painlevé paradox, European Journal of MechanicsA/Solids 21(5), 869 (2002)

32. Y. Or, E. Rimon, Investigation of Painlevé's paradox and dynamic jamming during mechanism sliding motion, Nonlinear Dynamics 67(2), 1647 (2012)

33. M. Erdmann, On a representation of friction in configuration space, The International Journal of Robotics Research 13(3), 240 (1994)

34. P. Flores, Concepts and formulations for spatial multibody dynamics (Springer, Berlin, 2015)

35. C.C.D. Wit, H. Olsson, K.J. Astrom, P. Lischinsky, A new model for control of systems with friction, IEEE Transactions on Automatic Control 40(3), 419 (1995)

36. J. Kövecses, Dynamics of mechanical systems and the generalized free-body diagram—Part I: general formulation, Journal of Applied Mechanics 75, 061012 (2008)

37. J. Kövecses, J.M. Font-Llagunes, An eigenvalue problem for the analysis of variable topology mechanical systems, Journal of Computational and Nonlinear Dynamics 4(3), 031006 (2009)

38. J.M. Font-Llagunes, A. Barjau, R. Pàmies-Vilà, J. Kövecses, Dynamic analysis of impact in swing-through crutch gait using impulsive and continuous contact models, Multibody system dynamics 28(3), 257 (2012) 